\title{
Siguiendo los pasos de Samuel Ramos: una propuesta metodológica de análisis de nuestras circunstancias
}

Mauricio Ávila Barba Universidad Autónoma de Querétaro

Resumen

En este trabajo replanteamos una de las preguntas más relevantes para los filósofos mexicanos de la primera mitad del siglo XX: ¿cómo hemos llegado a ser lo que somos? Para tal efecto, exponemos el análisis histórico-psicológico con el que Samuel Ramos diagnosticó el sentimiento de inferioridad que padece el mexicano. Empero, advertimos también que en este análisis Ramos mencionó otros casos que no fueron explicados por medio de sus presupuestos psicológicos; por lo que sugerimos que él los planteó -aunque no de manera explícita- en términos de condiciones de posibilidad, que habrían jugado un papel importante en la conquista del México prehispánico; un ejemplo de ello, es el egipticismo indígena. 
Nuestra propuesta consiste en analizar estos casos y problematizarlos por medio de la noción de condiciones de posibilidad de experiencia, la cual articulamos con los conceptos de valor y de sentido, de Gilles Deleuze, y con la ontología-histórica, de Michel Foucault. El objetivo de este trabajo es sugerir elementos metodológicos para analizar nuestras circunstancias y, así, hacernos cargo de la pregunta sobre cómo hemos llegamos a ser lo que somos. Con esto pretendemos seguir los pasos de Samuel Ramos, aunque, al mismo tiempo, nos distanciamos de él.

Palabras clave: sentido, valor, ontología-histórica, condición de posibilidad, experiencia.

\section{Abstract}

In this paper we reconsider the question: why we are that we are? The issue was important to the Mexican philosophers of the first half of the twentieth century. For this reason, we expose the historical-psychological analysis and the diagnosis of the inferiority complex that Samuel Ramos made about the Mexican. Nevertheless, in addition we describe some aspects that Ramos doesn't explain through in his physiological disquisitions. That's why we suggest that he formulated this aspects in terms of conditions of possibility, who were important to the conquest of Prehispanic Mexico; one example is the 'Indian egipticis'.

We analyze these cases using the notion of conditions of possibility of experience, that we define using another concepts, such as value and meaning (Gilles Deleuze) and Historical ontology (Michel Foucault). The aim of this paper is to suggest methodological guidelines for analyzing our circumstances and to analyze the issue: why we are that we are? In this way, we follow the path of Samuel Ramos, but, at the same time, we grow apart of his ideas.

Keywords: Meaning (direction), Value, Historical ontology, Condition of possibility, Experience. 
Me ha parecido siempre que una de las maneras de hacer filosofia mexicana es meditar sobre nuestra propia realidad filosófica, la de los filósofos y sus ideas, para averiguar si existen rasgos dominantes que pudieran caracterizar un pensamiento nacional.

Samuel Ramos

\section{Introducción: Nuestras circunstancias}

$\mathrm{D}$ esde las promesas de Felipe de Jesús Calderón Hinojosa, "el Presidente del empleo", pasando por la "Republica amorosa", de Andrés Manuel López Obrador, hasta el slogan "Por un México exitoso", de Enrique Peña Nieto, nos enfrentamos con un México que se reinventa y se "mejora" cada sexenio. Arrojados (estado de yecto) en este gran caldo de cultivo de proyectos de nación, los habitantes de este país (mexicanos y extranjeros) damos rumbo, con tumbos o con paso firme, según como se quiera ver, al porvenir de una República Mexicana que está signada por el encuentro de muchos mundos. ${ }^{1}$

Dos emperadores -Agustín de Iturbide y Maximiliano de Habsburgo-, 62 presidentes, 3 regencias -José Mariano Salas, Antonio de Labastida y Juan Bautista Ormachea-, algunas guerras - de Independencia, de Reforma-, distintas revoluciones -la de Villa, la de Madero, la de Zapata-, junto a las muchas reformas que actualmente se discuten y se aprueban con el motivo de un "Pacto por México", se postulan como el horizonte que delimita una realidad nacional que nos sale al paso, nos interpela y nos invita a una profunda meditación.

${ }^{1}$ El encuentro es un crisol en donde no sólo se funde el mundo español y el mundo indígena, también el francés, el chino, el norteamericano y muchos otros más de otra índole, como un mundo católico, uno protestante y, quizá en menor medida, uno judío. 
La pregunta sobre las circunstancias concretas que definen a México y la manera de hacerse cargo de ellas, delimitó una de las tareas que emprendieron filósofos mexicanos como Samuel Ramos, Emilio Uranga, Leopoldo Zea, entre otros. En diálogo con Ramón Xirau, con José Gaos, con José Ortega y Gasset, este grupo de jóvenes intelectuales entabló un análisis de la historia del pensamiento mexicano, aunado a una reflexión sobre su entorno. Así, para hacerse cargo de su situación -o para decirlo con Ortega y Gasset: para salvar nuestras circunstancias- era indispensable realizar un diagnóstico sobre cómo hemos llegado a ser lo que somos. Bajo este dictamen sería posible entender no sólo las flaquezas y las virtudes del mexicano sino, también, sus posibilidades reales (cfr. Ramos, 1934: 89-95). La misma tarea, que no implica necesariamente una suerte de regionalismo, es el motivo de este trabajo.

Ahora bien, en su momento, el horizonte hermenéutico que exigía la envergadura de esta empresa, la cual pretendía dar cuenta de nuestra realidad, se articuló con las Lecciones sobre la filosofía de la historia universal, de Georg Wilhelm Friedrich Hegel, las Meditaciones sobre el Quijote, de José Ortega y Gasset, el Ser y Tiempo, de Martin Heidegger, y la Introducción a las Ciencias del espiritu. En el que se trata de fundamentar el estudio de la sociedad y de la historia, de Wilhelm Dilthey. Así, la historia de la filosofía y la filosofía de la historia caracterizaron la escena de la filosofía en México, lo que, en alguna medida, sigue siendo así.

En su libro El perfil del hombre y la cultura en México, Samuel Ramos testificó la tarea histórica-psicológica que propuso para definir la mentalidad y la cultura del mexicano. Está muy documentado su diagnóstico: el sentimiento de inferioridad en éste. Sin embargo, creemos que en su análisis histórico Ramos nos remite a casos que no explica por medio de la psicología adleriana; por ejemplo, sus propuestas sobre el egipticismo indígena. En estos casos, Ramos hace referencia, aunque no de forma explícita, a cier- 
tas condiciones de posibilidad que habrían jugado algún papel en la conquista del mundo prehispánico, a saber: la religiosidad del indio mexicano, tierra fértil propicia para la recepción de la religiosidad española.

Nuestra propuesta es re-leer los casos expuestos anteriormente bajo la mirada de Michel Foucault y de Gilles Deleuze -ambos de vena nietzscheana-. ${ }^{2}$ Consideramos que por medio de sus lineamientos epistémicos, históricos y metodológicos, podemos proporcionar elementos para plantear y comprender las condiciones de posibilidad, sugeridas por Ramos y que nosotros llamaremos "condiciones de experiencia posible", que definen tanto las actitudes y las conductas individuales como las sociales de los mexicanos. Dicho esto, es claro que aunque seguimos a Ramos, pues nos pone en este camino, también nos alejamos de él.

Por lo tanto, nuestro objetivo es entonces sugerir elementos metodológicos para analizar nuestras circunstancias y, así, hacernos cargo de la pregunta sobre cómo hemos llegamos a ser lo que somos. Para tal efecto, este trabajo lo dividimos en dos apartados: en el primero, exponemos el análisis histórico-psicológico que Ramos realizó sobre el perfil del mexicano y también destacamos los casos que consideramos no tienen una explicación psicológica, los cuales nos sugieren realizar un análisis distinto al de Ramos; en el segundo, desarrollamos los aspectos histórico-metodológicos de Gilles Deleuze y de Michel Foucault, para destacar las nociones

${ }^{2}$ Estamos al tanto de los acalorados debates sobre la originalidad y la autenticidad de nuestra filosofía mexicana. Al respecto, considero que es importante señalar que, lejos de enfrascarme en una discusión sobre la originalidad del pensamiento mexicano y su dependencia con la filosofía europea, hago eco de las palabras de Gabriel Vargas Lozano, plasmadas en su artículo "El debate filosófico de los sesenta y su significación actual", en donde advierte que la filosofía auténtica -en sentido de propia-es aquella que contribuye a plantear y a proponer vías de solución a los problemas que afectan nuestra realidad; lo cual no implica un regionalismo filosófico (Lozano, 2007: 171). 
de "valor" y de "sentido" y, así, definir la idea de "condiciones de experiencia posible"; después, articulamos estas tres nociones con la ontología-histórica foucaultiana.

\section{Seguir los pasos de Samuel Ramos: pensar las condiciones de posibilidad del mexicano}

En el libro El pensamiento mexicano del siglo XX, Abelardo Villegas señaló que la Revolución de 1910 en México fue una coyuntura que dio paso a la transformación de la mentalidad de los mexicanos. Todo un acontecimiento moral, cultural y mental que planteó una reivindicación laboral, educativa, agraria y económica, entre otras (cfr. Villegas, 1993: 93). Villegas advierte que este acontecimiento orientó el rumbo de la reflexión filosófica en México hacia una tarea de autognosis -algo más que la mera autoconciencia-. Esta empresa fue la impronta que seguirían, a su modo y desde diferentes presupuestos, Leopoldo Zea (cfr. 1974: IX), Emilio Uranga (cfr. 1952: 9-16) y, desde la literatura, Octavio Paz (cfr. 1992: 12); lo cual delinearía, según Abelardo Villegas, un nacionalismo filosófico en México (cfr. Villegas, 1993: 145-146).

Bajo las circunstancias de una nación independiente que "florecía" después de la agitación de las distintas revoluciones, en el libro Historia de la filosofía en México, Samuel Ramos dirigió el rumbo de su reflexión filosófica a la labor de precisar nuestra personalidad en formación. Para él, esto significó una tarea por especificar la definición "de la circunstancia mexicana, la determinación de lo que es o puede ser su cultura, tomando en cuenta las modalidades propias de nuestra historia y la forma en que éstas han moldeado la fisionomía peculiar del hombre mexicano" (Ramos, 1943: 222).

El método -el camino- que Ramos siguió en esta investigación, quedó expuesto en el primer apartado de su obra el Perfil del hombre y la cultura en México. La encomienda fue partir de las realida- 
des efectivas para definir la cultura mexicana mediante el análisis de la estructura mental del hombre y los accidentes de su historia. Bajo estos supuestos, Ramos elaboró una filosofía de la cultura y una psicología que explicaban las modalidades del ser del mexicano. Se sirvió de la articulación entre la psicología de Alfred Adler y la historia concreta del mexicano, desde la Conquista hasta sus días, para dar cuenta de cómo los mexicanos, que estaban gestando un joven país, se vieron enfrentados, real y psicológicamente, a las circunstancias de un México independiente que atravesaba por una post-revolución.

Desde los supuestos de Adler, Ramos propuso que la desproporción que existe entre lo que uno quiere hacer y lo que se puede hacer, conducía a un sentimiento de incapacidad y de desconfianza de sí mismo; si es mayor lo que se quiere hacer de lo que se puede hacer, el resultado es obvio. Desde esta perspectiva, como señaló Guillermo Hurtado, Ramos explicó las actitudes y la conducta individual y social de los mexicanos. Las circunstancias concretas de México y su cultura derivada de Europa, explican la causa del sentimiento de incapacidad y de desconfianza de sí mismo, en el mexicano. Al respecto, Hurtado señaló que "la causa de ese complejo es que los mexicanos [se] midieron con valores que les quedaban muy altos en un momento de su desarrollo histórico [,] en el que tenían que haber sido más modestos en sus aspiraciones" (Hurtado, 2012: 61). En este sentido, Ramos señaló que:

el sentimiento de inferioridad en nuestra raza tiene un origen histórico que debe buscarse en la conquista y colonización. Pero no se manifiesta ostensiblemente sino a partir de la independencia, cuando el país tiene que buscar por sí solo una fisionomía nacional. Siendo un país joven, quiso, de un salto, ponerse a la altura de la vieja civilización europea y entonces estalló el conflicto entre lo que se quiere y lo que se puede (Ramos, 1934: 91). 
Son muy conocidas las figuras en donde el sentimiento de inferioridad, como un síntoma, se hace patente. Tres ejemplos permiten a Ramos dibujar una fisionomía nacional: el pelado mexicano, con su hombría quimérica; el mexicano de la ciudad, con su desconfianza irracional, y el burgués mexicano, con su vergüenza de ser mexicano. Aunque cabe destacar que este sentimiento de inferioridad no es un mal esencial o intrínseco, ya que deriva, como se dijo, de la historia del mexicano.

Para Ramos, todas estas figuras se sustentan en valoraciones impropias y en sobreestimaciones que falsean la realidad. El "pelado" que busca rińa como un excitante para elevar el tono de su "yo" deprimido, el "mexicano de la ciudad" que desconfía del mundo y de los hombres y el "burgués mexicano" que toma como empresa el construir su propia imagen conforme a un deseo de superioridad, son sólo posturas -ardides- que carecen de todo valor real. Con este diagnóstico, el mexicano se podría comprender como "un hombre que huye de sí mismo para refugiarse en un mundo ficticio. Pero así no liquida su drama psicológico" (Ramos, 2001: 64).

Como señala Guillermo Hurtado, Ramos pensó "que una concepción objetivista de los valores, como las de Scheler y Hartmann, sería de utilidad para que los mexicanos superaran los vicios morales" (Hurtado, 2012: 65). Ramos nos invitó entonces a una reflexión ética y educativa para reencontrar el humanismo y los valores reales de México; sin que esto signifique necesariamente la apuesta por un provincialismo. Para ello, él nos encomendó realizar una reflexión que no se encerrara ni en un europeísmo ni en un falso mexicanismo puro, para poder dirigirnos hacia a un nuevo humanismo que se caracterizara por una conciencia más justa, más documentada de los valores -reales- del hombre (Ramos, 1962: 37ss.) Esta empresa se cristalizaría, según Ramos, con la sistematización de la educación, que estaría dirigida al cultivo de la lengua 
y de la literatura, como una base en la que se asentaría la cultura nacional, y debería acompañarse de la historia, la geografía, las ciencias naturales, así como de la vida y la filosofía (Ramos, 2001: 115-116).

Si bien Ramos nos mostró una vía de análisis histórico-psicológico para responder a la pregunta sobre cómo llegamos a ser lo que somos y desde ahí plantear las condiciones bajo las cuales se podría dar rumbo a este naciente país - un nuevo humanismo y una sistematización de la educación-, también, desde nuestra perspectiva, en el Perfil del hombre y la cultura en México, nos sugirió otros presupuestos desde su análisis histórico del ser del mexicano. Es a partir de estas implicaciones donde coincidimos con él y también nos distanciamos. Pongamos algunos ejemplos de estos casos.

Ramos advirtió que la implantación del espíritu español en América se llevó a cabo en dos etapas: una de trasplantación y otra de asimilación. Nos quedaremos en el primer momento. El proceso de trasplantación tuvo como vehículo la imposición del idioma castellano y de la religión católica en el nuevo mundo. Los ejecutores de esta tarea fueron los misioneros que realizaron, en el siglo XIX, la conquista espiritual de México (cfr. Ramos, 1934: 102). Sin embargo, él también sugirió que la obra de los misioneros -que realizaron la trasplantación de la cultura española en México- "fue facilitada por cierta receptividad de la raza aborigen, que era tan religiosa como la del hombre blanco que venía a dominarla. Era un terreno muy bien preparado para que la semilla cristiana prendiera en el nuevo mundo" (Ramos, 1934: 103). Asimismo, en su análisis sobre "el egiptisimo indígena", Ramos hizo aseveraciones que, quizá, hoy nos parecerían impensables y arriesgadas. Por ejemplo, él no creyó que "la pasividad del indio fuera exclusivamente un resultado de la esclavitud en la que cayó al ser conquistado. Se dejó conquistar tal vez porque ya su espíritu estaba dispuesto a la pasividad" (Ramos, 2001: 36); característica 
que advirtió en el arte indígena. Además, indicó que "desde antes de la conquista los indígenas eran reacios a todo cambio, a toda renovación. Vivían apegados a sus tradiciones, eran rutinarios y conservadores. En el estilo de su cultura quedó estampada la voluntad de lo inmutable" (Ramos, 2001: 36). Por esta razón, para Ramos, al indio de México (sin ser inferior al europeo), por su egipticismo, le fue imposible -o difícil- asimilar una cultura que se regía por una ley del devenir, del cambio. ${ }^{3}$

Con estos ejemplos queremos destacar las características que, según Ramos, habrían condicionado la conquista -o el encuentro entre dos mundos- y nuestra historia. Sin embargo, también queremos hacer notar que esta interpretación del indio mexicano no está articulada bajo los presupuestos de la psicología de Adler. Al mismo tiempo, enfatizar que si bien Ramos advirtió que el egiptisimo indígena era algo propio de ellos - del indio mexicano- no lo asumió como una característica esencial. Desde nuestra perspectiva, Ramos planteó el egiptisimo indígena en términos de una condición de posibilidad de la conquista. Pongamos otro ejemplo y apelemos a datos duros.

En su documento de trabajo Desigualdad de oportunidades y politicas públicas en México: el fracaso del proyecto redistributivo, John Scott advirtió que según encuestas del Comité Técnico para la Medición de la Pobreza, realizado en el 2002, se encontró que mucha gente pobre explica su condición de indigencia apelando a la voluntad de Dios: "así lo quiere Él” (cfr. Scott, 2005: 3). La razón de esto podría plantearse desde muchas perspectivas como

${ }^{3}$ Aquí no nos metemos en la discusión sobre si la concepción ramosiana de la cultura es, como advirtió Gabriel Vargas Lozano, unilateral. Desde esta perspectiva, Ramos no valoró adecuadamente la cultura indígena, la cual fue subordinada (Varga, 2005: 202). Aunque este problema es interesante, no es relevante para el objetivo de este trabajo. 
las religiosas, las psicológicas o las antropológicas. ${ }^{4}$ Por mi parte, quisiera plantear lo anterior en términos de las condiciones de posibilidad. En otras palabras, así como Ramos propuso que el nuevo mundo era una tierra fértil -como condición- para la recepción de la religiosidad española y gracias a ello y otras circunstancias fue posible la conquista espiritual del México prehispánico, nosotros queremos destacar que explicar la indigencia apelando a la voluntad divina delimita también ciertas condiciones, desde las cuales podrían abrirse muchas -infinitas- posibilidades de ser del mexicano. 5 Por lo tanto, es relevante reflexionar en la sugerencia que Samuel Ramos deja en el aire. Quizá las condiciones que definen nuestra experiencia y expectativas de desarrollo, en cierta medida, delimitan la posibilidad o la imposibilidad de propuestas políticosociales concretas, llámese equidad social, política de salud, entre otras. ${ }^{6}$ Es aquí, desde nuestra perspectiva, que las propuestas de Gi-

${ }^{4}$ Por ejemplo, desde una perspectiva antropológica, en su texto La antropología del hecho religioso, José María Barrio Maestre señaló que "en cierto modo, la experiencia religiosa tiene que ver con la percepción de que el mundo no puede explicarse por sí mismo... La religión muestra el hombre, en todo tiempo y lugar, enfrentado con un destino que trasciende su propia muerte, destino con el que ha de armonizar su existencia terrena" (2012: 38).

${ }^{5}$ Quiero aclarar que podríamos hacer eco de la sentencia kantiana que articula su opúsculo “¿Qué es la Ilustración?”: ¡SAPERE AUDE! (Atrévete a pensar por ti mismo) y atribuir una ignorancia, que podría salvarse con la educación, a los individuos que explican religiosamente los sucesos de su vida. Sin embargo, esto no lo cuestionamos. Nos importa, como ya mencionamos, entender esa religiosidad como una condición.

${ }^{6}$ Por poner otros ejemplos, hay que destacar que José Amado Aguirre ha puesto en evidencia la vena cristiana que hay el marxismo que se considera ateo (cfr. Amado, 2010); o bien el de la deconstrucción del cristianismo que están llevando a cabo intelectuales franceses, como René Girad, Jean-Luc Nancy o Michel Onfray, en donde se ha puesto en evidencia la herencia cristiana en la formación de estado laico (Girard y Vattimo, 2011). Estas propuestas definen cierto horizonte de comprensión y de formación de nuestras circunstancias, que podría pensarse como una condición de posibilidad. 
lles Deleuze y la ontología-histórica de Michel Foucault podría darnos luces al respecto.

\section{La revolución copernicana y la ontología-histórica}

Los textos de Friedrich Nietzsche son huesos muy duros de roer: por medio de ellos puede verse al "maestro de la sospecha" -como lo recuerda Jacques Le Rider (1999), en su libro Nietzsche en France, de la fin du XIX siècle au temps présent- o al más grande teólogo después de San Pablo -según el antropólogo René Girard (2011)-. Nuestra lectura se carga a la primera propuesta y dejamos pendiente la discusión sobre la segunda.

En su libro Nietzsche et la philosophie (1983), Gilles Deleuze expone de manera clara cuál es la postura crítica que Nietzsche hereda a la historia de la filosofía: la intromisión del 'valor' y del 'sentido', como instancias nodales para dilucidar la noción de 'fenómeno' y, en todo caso, para hacer el análisis de un discurso. Para

\footnotetext{
${ }^{7}$ Varias cosas hay que señalar al respecto. Por un lado, debemos recordar grosso modo que el pensamiento de la sospecha se colocó sobre el campo general de los enunciados humanos, pues éstos dicen siempre otra cosa de lo que creen decir: enunciado de la voluntad de poder, según Nietzsche, del inconsciente, según Freud, y de la lucha de las clases y de las relaciones de producción, según Marx. Por otro lado, debemos advertir que el análisis sobre la noción de 'valor', tiene una tradición que se remonta, al menos, a los moralistas franceses como Montaigne y Voltaire, entre otros. Herbert Frey expuso el vínculo de Nietzsche con Montaigne, en su artículo "Nihilismo y arte de la vida. Entre Montaigne y Nietzsche", y destacó la importancia de las nociones de 'valor' y de 'sentido' que articulan el nihilismo nietzscheano (cfr. Frey, 2011). Finalmente, también resulta interesante señalar que en México dicho análisis no fue ajeno a intelectuales como Antonio Caso (cfr. 1943), Samuel Ramos (cfr. 1928) o como Octavio Paz, quien declara que El laberinto de la soledad -obra en donde se pregunta cómo el mexicano ha llegado ser lo que es- se inscribe en la tradición de los moralistas franceses que "trataron de describir, de un modo crítico, el temperamento (la moral) de los hombres" (cfr. Paz, 1991).
} 
Herbert Frey, traductor y crítico de Nietzsche, con el nihilismo se daba cuenta de la carencia objetiva de valor y de sentido del mundo y del hombre; sin embargo, también advierte que "con ello, empero, no quería decir que no podían existir creaciones de valor y de sentido, sino sólo que las propiedades de valor del mundo y del hombre no estaban fundamentadas en sí mismas, sino en valoraciones subjetivas de los seres humanos" (Frey, 2011: 39). Así, como apunta Deleuze, el concepto de valor implicó una inversión crítica: "los valores aparecen o se ofrecen como principios: una valoración supone valores a partir de los cuales ésta aprecia los fenómenos" (1983: 2).

En palabras de Gilles Deleuze, esto significó una inversión crítica en el conocimiento y en la forma de concebir nuestra experiencia del mundo. En palabras llanas, si con el empirismo de David Hume la experiencia del mundo se articulaba desde la noción de 'percepción' (cuya derivación eran las ideas y las impresiones), o si con el criticismo de Kant la experiencia posible del mundo estaba en dada en términos de condiciones a priori de la sensibilidad y categorías a priori del entendimiento, entonces con Deleuze -por medio de Nietzsche- el conocimiento estará planteado en términos de 'valor'.

No es de extrañar -sugiere Deleuze- que para Nietzsche el conocimiento tenga un origen mezquino. En su obra Sobre verdady mentira en sentido extramoral, Nietzsche afirmó que:

En algún apartado rincón del universo centelleante, desparramado en innumerables sistemas solares, hubo una vez un astro en el que animales inteligentes inventaron el conocimiento.

¿Qué es, pues, verdad? Respuesta: una multitud movible de metáforas, metonimias y antropomorfismos, en una palabra una suma de relaciones humanas poética y retóricamente potenciadas, transferidas y adornadas que tras prolongado uso se le antojan fijas, canónicas y obligatorias a un pueblo. Las verdades son ilu- 
siones que se han olvidado que lo son, metáforas gastadas cuya virtud sensible se ha deteriorado, monedas que de tan manoseadas han perdido su efigie y ya no sirven como monedas, sino como metal (1996:17).

Si es así, si el conocimiento es un invento vinculado con el valor, se puede advertir "que Kant no realizó la verdadera crítica, porque no supo plantear el problema [del conocimiento] en términos de valores" (Deleuze, 1983: 1). En todo caso, tendríamos que pensar que las verdaderas condiciones de nuestra experiencia no están dadas por las formas a priori de la sensibilidad (espacio y tiempo) ni por categorías a priori del entendimiento, sino en por el valor. Desde esta perspectiva, todo fenómeno humano nos es dado en su sentido si desentrańamos los valores y valoraciones que lo significan; en otras palabras:

si sabemos cuál es la fuerza que se apropia de la cosa, que la explota, que se apodera de ella o se expresa en ella. Un fenómeno no es una apariencia ni tampoco una aparición, sino un signo, un síntoma que encuentra su sentido en una fuerza actual. Toda la filosofía es una sintomatología y una semiología. Las ciencias son un sistema sintomatológico y semiológico (Deleuze, 1983: 3).

La tarea de una filosofía crítica, de una filosofía a martillazos como lo expresa el propio Nietzsche-, "tiene dos movimientos inseparables: referir cualquier cosa, y cualquier origen de algo a los valores; pero también referir estos valores a algo que sea como su origen, y que decida su valor" (Deleuze, 1983: 2). Bajo estos supuestos, la genealogía sería la ruta crítica pertinente para advertir la pluralidad de sentidos, efectos de los valores, como signos de aquella fuerza que se apodera de la cosa. Sin embargo, como lo hizo notar Michel Foucault en su artículo "Nietzsche, la généalogie, l'histoire", Hommage à Jean Hyppolite", debemos ser muy 
cuidadosos al hablar sobre el origen. Cuando Nietzsche hace referencia a esta noción,

rechaza, al menos en ciertas ocasiones, la búsqueda del origen (Ursprung). Porque en primer lugar [la búsqueda del origen] se esfuerza por recoger allí la esencia exacta de la cosa, su más pura posibilidad, su identidad cuidadosamente replegada sobre sí misma, su forma móvil y anterior a todo aquello que es externo, accidental y sucesivo. Buscar un tal origen, es intentar encontrar "lo que estaba ya dado", lo "aquello mismo" de una imagen exactamente adecuada a sí (Foucault, 1971: 136).

En este sentido, Foucault advierte que todo trabajo que quisiera, por ejemplo, dar cuenta del 'origen del mal', como en su momento lo pretendió Paul Ree, en su obra Ursprung der moralischen Empfindungen, tendría que regresar hasta la fuente de dónde el propio mal emana. Una genealogía de la moral tendría que enfocarse en esta tarea.

Por el contrario, para Foucault detrás de las cosas -si se me permite la expresión, pues él no es un estructuralista- no hay un secreto esencial y sin espacio; su único secreto es que ellas no tienen esencia o, en todo caso, ésta fue edificada (construite) por piezas que le son extrañas. En este sentido,

Hacer la genealogía de los valores, de la moral, del ascetismo, del conocimiento no será por tanto partir a la búsqueda de su "origen”, minusvalorando como inaccesibles todos los episodios de la historia; será por el contrario ocuparse en las meticulosidades y en los azares de los comienzos; prestar una escrupulosa atención a su derrisoria malevolencia; prestarse a verlas surgir quitadas las máscaras, con el rostro del otro; no tener pudor para ir a buscarlas allí donde están "revolviendo los bajos fondos"-, dejarles el tiempo para remontar el laberinto en el que ninguna verdad nunca jamás las ha mantenido bajo su protección (Foucault, 1971: 137). 
Una genealogía, que hace suya la verdadera revolución copernicana, se dirigiría al origen del valor, lo que significa: estar a la caza de los eventos (quiebres) de la "la historia, con sus intensidades, sus debilidades, sus furores secretos, sus grandes agitaciones febriles y sus síncopes, [que] es el cuerpo mismo del devenir" (Foucault, 1971: 137).

A pesar de ello, no hay que engañarnos, ni Foucault ni Nietzsche ni Deleuze están a la búsqueda de La historia. El horizonte hermenéutico de Foucault se abre en los pliegues, las fisuras, los quiebres de las historias regionales (una historia de la locura, una historia de la clínica, etc.) y no se despliega en una historia que se remonta a una suerte de primera causa de efecto universal, que unifica y da consistencia -como una mezcla homogénea entre lo idéntico y lo nuevo- al pasado y al presente. En congruencia con lo anterior, pero desde diferentes supuestos, Deleuze advierte que "la historia es la variación de los sentidos, es decir la sucesión de los fenómenos de sujeción más o menos violentos, más o menos independientes unos de otros. El 'sentido' es pues una noción compleja: siempre hay una pluralidad de sentidos, una constelación, un conjunto de sucesiones pero también de coexistencias, que hace de la interpretación un arte" (Deleuze, 1983: 4).

El giro copernicano que, según Deleuze, Nietzsche introdujo al ámbito del conocimiento y de la historia, por medio de las nociones de 'valor' y de 'sentido', nos pone ante las condiciones que determinan nuestra experiencia del mundo y nuestras posibilidades de ser; además, traza una ruta de análisis de nuestras circunstancias y, con ello, permite replantear la pregunta: ¿cómo hemos llegado a ser lo que somos?, qué tanto interesó a Samuel Ramos y a otros filósofos mexicanos. A esto nos referíamos cuando, en el primer apartado de este trabajo, enfatizamos un punto seńalado por Ramos, donde habla sobre cómo la obra de los misioneros -que 
realizaron la trasplantación de la cultura española a México- fue facilitada por cierta receptividad de la raza aborigen, que era tan religiosa como la del hombre blanco, y, también, cuando nos referimos a su análisis sobre el egipticismo indígena. Desde nuestra perspectiva, Ramos está señalando ciertas condiciones de posibilidad y de experiencia de los indios mexicanos, que habrían jugado un papel importante en la conquista, y que, desde nuestra perspectiva, podríamos analizar en términos de 'valor' y de 'sentido'. ${ }^{8}$ Sin embargo, queremos dar un paso adelante. Ahora nos gustaría plantear estas condiciones ya en el ámbito de la ontología-histórica foucaultiana, la cual podemos tomar, guardando toda proporción, como una continuación de lo expuesto por Nietzsche y Deleuze. Para esto, permítasenos poner otro ejemplo sobre cómo pensar las

${ }^{8}$ Aquí dejamos otro ejemplo, aunque desde otros presupuestos. En su libro Multiculturalismo y Pluralismo, León Olivé analizó el conflicto zapatista, en el marco del diálogo que se entabló entre éstos y las autoridades federales de la República Mexicana. Un encuentro que, por cierto, responde a los valores que definen a una sociedad democracia. El diagnóstico de Olivé es muy sugerente para nuestro análisis. En primer lugar, él destacó los grandes abismos lingüísticos que existen entre la comunidad hispanohablante y los miembros de las comunidades indígenas de Chiapas; lo que implica, a decir de Olivé, una diferencia ontológica entre los mundos y la experiencia de ellos. Al respecto, por medio del lingüista Carlos Lenkersdorf, Olivé señaló que los estudios de éste: "En los que explica que una sencilla oración como "(lo) dije, ustedes (lo) escucharon", no admite una traducción exacta al tojolabal... Esta oración se refiere a un hecho al que ninguna frase en tojolabal puede referirse. [El] hecho al que alude la frase en español ...es imposible de reconocer desde la visión del mundo de los tojolabales... Pero el hecho existe en virtud de la estructura misma de la lengua española. Así, no es que los tojolabales no la puedan reconocer, sino que eso no existe como hecho en su mundo" (Olivé, 2007: 109). Con ello Olivé destacó que las diferencias lingüísticas no presentan simples problemas de traducción. Al contrario, con ello están en juego distintas formas de comprender, de experimentar y de interactuar con la realidad. Así, Olivé advierte que "las comunidades indígenas de Chiapas y los hispanohablantes de las sociedades modernas viven en mundos diferentes (Olivé, 2007: 110). 
condiciones de posibilidad de experiencia: en Las palabras y las cosas. Una arqueología de las ciencias humanas, Michel Foucault expuso un caso límite del pensar, de experimentar el mundo. Al respecto, nos recuerda que en un libro de Borges, en el que se expone "cierta enciclopedia china" donde:

los animales se dividen en $a$ ] pertenecientes al Emperador, $b$ ] embalsamados, $c$ ] amaestrados, $d]$ lechones, $e$ ] sirenas, $f$ fabulosos, $g$ ] perros sueltos, $h$ ] incluidos en esta clasificación, $i$ ] que se agitan como locos, $j$ ] innumerables, $k$ ] dibujados con un pincel finísimo de pelo de camello, $l$ etcétera, $m$ ] que acaban de romper el jarrón, n] que de lejos parecen moscas (Foucault, 2009: 9).

Foucault advierte que con este 'canto exótico' se muestra una imposibilidad del pensar, un límite o una experiencia que no es posible en nuestro mundo. Para repensar estos límites -y posibilidades-, Foucault partió de un supuesto epistémico que, en un primer momento, determinó el camino de su historia crítica del pensamiento: afirmó que el ámbito del pensamiento debe entenderse como un acto que correlaciona, en una diversidad infinita de vínculos posibles, a un sujeto y a un objeto. Por lo tanto, la tarea de una historia crítica del pensamiento tendría que enfocarse al "análisis de las condiciones en las que se han formado o modificado ciertas relaciones entre sujeto y objeto; y ello en la medida en que tales relaciones son constitutivas de un saber posible" (cfr. Foucault, 1984: 942). Desde esta perspectiva, se trataría entonces de determinar los modos de subjetivación, para indagar cómo se ha constituido un sujeto del saber, y de objetivación, para indagar cómo se ha constituido un objeto de la ciencia, que precisamente ha sido considerado como digno de ser problematizado como un objeto de conocimiento. Finalmente, a esta relación entre sujeto y objeto, Foucault ańade los 'juegos de verdad', que no significa: 
el descubrimiento de las cosas verdaderas, sino [de] las reglas según las cuales, y a propósito de ciertas cosas, lo que un sujeto pueda decir queda sometido a la pregunta por lo verdadero o lo falso.

[...] la historia crítica del pensamiento no es ni una historia de las adquisiciones ni una historia de los ocultamientos de la verdad. Es la historia de la emergencia de los juegos de verdad; es la historia de las "veridicciones" (veridictions) entendidas como las formas según las cuales se articulan, en un cierto dominio de cosas, discursos susceptibles de ser enunciados como verdaderos o como falsos (Foucault, 1996: 633).

Además de este principio epistémico -el acto de correlacionar un sujeto y un objeto-, en su historia crítica del pensamiento, Foucault quiso hacer eco de un reto lanzado por Kant en el marco de la Ilustración. En su artículo “Qu'est-ce que les Lumières?”, Foucault advierte una pregunta que resuena en la historia de la filosofía a partir de Kant: ¿cuál es, entonces, este evento que denominamos Aufklärung y que ha determinado, al menos parcialmente, lo que somos, lo que pensamos y lo que hacemos hoy día? Esta cuestión requiere, necesariamente, ponerse al día sobre la significación del reto que lanza Kant y que habría delineado todo el proyecto de la modernidad, puesto que de "Hegel a Horkheimer, o a Habermas, pasando por Nietzsche o por Max Weber, apenas hay filosofía que, directa o indirectamente, no se haya visto enfrentada a esta misma pregunta" (Foucault, 1993: 7). En palabras de Miguel Morey, la labor de repensarse bajo la guía de la pregunta ¿quién soy yo? en este momento de la historia -distinto al 'yo' cartesiano que es universal pero no histórico- dirigió a Foucault a una tarea por "dirimir el sentido y el valor de las cosas que (nos) pasan en nuestro presente" (1989: 23).

Con la ontología-histórica, Foucault enfrentó el reto lazado por Kant desde tres perspectivas, que no deben verse como excluyentes: 
-[Una] ontología histórica de nosotros mismo en relación a la verdad que nos constituye como sujetos de conocimiento.

-[Una] ontología histórica de nosotros mismos en las relaciones de poder que nos constituyen en cuanto actuando sobre los demás. -[Una] ontología histórica de nosotros mismos en relación ética por medio de la cual nos constituimos como sujetos de acción moral (Morey, 1989: 25).

Permítasenos delinear un caso más con el fin de exponer de qué trata la ontología-histórica foucaultiana. Para tal efecto, retomaremos al psicoanálisis. ${ }^{9}$ Con esto sugerimos cómo, por medio de la propuesta foucaultiana, podríamos repensar las condiciones de posibilidad de experiencia y, en este sentido, retomar la pregunta sobre cómo hemos llegado a ser lo que somos.

Siempre ha sido interesante el modo en el que el psicoanálisis se ha ido institucionalizando en algunos países, sobre todo en los llamados occidentales. Elisabeth Roudinesco, historiadora del psicoanálisis, en su artículo “¿Está superado el psicoanálisis?”, elaboró con mucha claridad un mapa de la difusión de éste por diferentes países. Algo muy importante a destacar es que Roudinesco sugiere condiciones políticas y sociales que posibilitan el desarrollo del psicoanálisis. Así, ella advirtió que:

para la implantación de las ideas freudianas y la formación de un movimiento psicoanalítico deben cumplirse dos condiciones. Primero, la constitución de un saber psiquiátrico, es decir, una mirada sobre la locura capaz de conceptualizar la noción de enfermedad mental en detrimento de la idea de posesión divina, sagrada o demoníaca. Y, en segundo término, la existencia de un

\footnotetext{
${ }^{9}$ No está de más aclarar que, si bien traemos a colación al psicoanálisis, no pretendemos refutar la propuesta sobre la psicología del mexicano que Ramos fincó en los presupuestos psicológicos de Alfred Adler.
} 
Estado de derecho capaz de garantizar el libre ejercicio de una enseñanza freudiana. Un Estado de derecho se caracteriza por los límites que pone a su poder sobre la sociedad y los ciudadanos, y por la conciencia de que tiene límites. Sin él, el psicoanálisis no puede ejercerse libremente, transmitirse por la cura o enseńarse en instituciones específicas. En otras palabras, toda implantación del psicoanálisis pasa por el reconocimiento consciente de la existencia del inconsciente, así como la asociación libre, como la técnica de la cura, pasa por el principio político de la libertad de asociación (Roudinesco, s/a).

En congruencia con lo anterior, Roudinesco señaló que en lugares como la China comunista, la India o la España del franquismo, el psicoanálisis no tuvo un desarrollo importante. Por el contrario, uno de los casos paradigmáticos de la implantación e institucionalización del psicoanálisis, es Argentina.

En una entrevista para la revista online Letra Urbana, el psicólogo Alejandro Dagfal advirtió que las costumbres y el lenguaje del argentino están fuertemente vinculados al psicoanálisis. Además, debemos destacar que, en congruencia con lo que apuntó Roudinesco, Dagfal señaló que el psicoanálisis en Argentina se vio bloqueado por los golpes militares de 1966 y 1976, por lo que sólo pudo desarrollarse bajo regímenes democráticos (cfr. Dagfal, s/a). Por otro lado, la importancia del psicoanálisis en Argentina fue señalada también por el periódico de derecha Clarín. En este diario se advirtió la tendencia de los porteńos a psicoanalizarse: "Mil pesos gasta por ańo, en promedio, una persona que va dos veces por semana a terapia, según estadísticas de la Asociación Psicoanalítica Argentina (APA). Y 154 psicólogos cada 100.000 habitantes es la proporción en la Argentina, cifra que representa el triple de la que hay en los Estados Unidos" (2012). Consideramos entonces que este país sureño constituye un caso significativo, no sólo para entender la institucionalización y el desarrollo del psicoanálisis sino, 
además, para evidenciar condiciones de experiencia posible, al menos, de los porteños.

Explicitemos la ontología-histórica para el caso del psicoanálisis. Desde la primera perspectiva de la ontología-histórica, realizaríamos un análisis de las condiciones en las que se han formado o modificado ciertas relaciones entre un sujeto y un objeto, en la medida en que tales relaciones son constitutivas de un saber posible. Desde esta perspectiva pensaríamos al psicoanálisis como un saber que estaría definido bajo cierta relación entre un sujeto y un objeto y habría sido posible en un momento de la historia, y que implicaría la emergencia de un juego de verdad, con el cual se articularían, en un cierto dominio de cosas, discursos susceptibles de ser enunciados como verdaderos o como falsos. ${ }^{10}$

Ahora bien, desde la segunda perspectiva de la ontología-histórica, habría que señalar que los saberes implican prácticas divisorias. Por ejemplo: lo sano y lo enfermo, lo normal y lo anormal, entre otras divisiones. Finalmente, desde la tercera perspectiva de la ontología-histórica, la cuestión apunta a interrogarnos por el "modo en el que el sujeto hace la experiencia de sí mismo en un juego de verdad en el que está en relación consigo mismo" (Foucault, 1984: 633). Sirva lo siguiente como un ejemplo: desde ciertos saberes podríamos concebirnos y hacer experiencia de nosotros como personas reprimidas. Desde esta perspectiva, Foucault nos encomienda no preguntarnos “¿por qué somos unos reprimidos?, sino ¿por qué decimos con tanta pasión, con tanto rencor contra nuestro pasado más próximo, contra nuestro presente y contra nosotros mismos que somos reprimidos?” (1986: 15-16). En términos llanos: ¿por qué hacemos experiencia de nosotros mismos, en tanto reprimidos?

${ }^{10}$ Aquí no se discute, como en su momento lo hizo Karl R. Popper, la cientificidad del psicoanálisis, pero sí sus condiciones históricas de emergencia. 
A menos que uno quiera aprender a ser psicoanalista y sólo se esté interesado en la teoría y en la técnica psicoanalítica, particularmente consideramos una tarea pertinente el interrogase por los juegos de valor y de sentido que están tras esta recurrencia al psicoanalista, al menos, de los porteńos. Esta práctica alimenta todo un saber que soporta un tipo de relación entre un sujeto y un objeto, que define a este saber y a la verdad que está en juego con él. De esta forma, en palabras de Foucault, es necesario analizar los discursos bajo la mira de entender "la voluntad que los mueve y de la intensión estratégica que los sostiene” (Foucault, 1986: 15). Finalmente, si apelamos a la revolución copernicana descrita por Deleuze, podemos decir que el saber psicoanalítico y la verdad que se encierra en él, constituyen una condición de posibilidad de la experiencia de mundo, al menos, de los porteños. Valga agregar que quizá no sea lo mismo para los mexicanos, de quienes se dice que están más vinculados a la confesión con un sacerdote o, como apunta Octavio Paz, a emborracharse para confesarse (1992: 21).

\section{Conclusiones}

¿Cómo seguir entonces los pasos y las tareas abiertas por Samuel Ramos? Debemos señalar que es obvio que el análisis históricopsicológico, que realizó este pensador sobre el mexicano, no puede pensarse como una ontología-histórica, a la manera de Michel Foucault. Esto se sigue por el simple hecho de que a aquél no le preocupó preguntarse por la constitución de la psicología, en la cual apoyó su diagnóstico del mexicano, y entender a ésta como un saber que surge bajo condiciones determinadas, el cual implica prácticas divisorias y formas en que el sujeto hace la experiencia de sí mismo.

Sin embargo, Samuel Ramos sí nos invita a reconocer los eventos que han sacudido la historia de México, aquellos que han dado 
sentido a lo que somos y en donde se muestran nuestras posibilidades. Ramos nos encomienda realizar una tarea titánica: reconstruir la historia de México, no sólo por medio de aquellos momentos que han significado y sacudido a este país sino, además, reconociendo las ideas filosóficas de "humanistas, hombres de ciencia, políticos, educadores, moralistas, etcétera” (1934: 101), que nos han definido como nación.

La pregunta por el cómo es que llegamos a ser lo que somos y, en este sentido, sobre cuáles son nuestras condiciones de experiencia posible del mundo, tiene una relevancia filosófica fundamental. A partir de la respuesta que obtuvimos de ella, se pueden poner en evidencia aspectos epistémicos, éticos -costumbre-, incluso, políticos y sociales, que nos determinan. Así, sin ser una ontología-histórica como la propuesta de Michel Foucault, el trabajo de Samuel Ramos nos invita con su pertinencia a repensar las condiciones de nuestra experiencia, de nuestras formas de subjetivación y de objetivación -para decirlo con Foucault- o de los juegos de 'valor' y de 'sentido' en la historia -para decirlo con Deleuze-.

Con Michel Foucault y Gilles Deleuze encontramos entonces un camino -método- para seguir con la tarea encomendada por Samuel Ramos: interrogarnos sobre cómo hemos llegado a ser lo que somos. La respuesta, como se vio, exige ir en búsqueda de los sucesos de la historia, de las sacudidas, de las sorpresas, de las victorias afortunadas y de las derrotas mal digeridas, que dan cuenta de los comienzos, de los atavismos y de las herencias (Foucault, 1971: 137). 


\section{Bibliografía}

Amado Aguirre, José, 2010, El cristianismo subyacente en el marxismo, Buenos Aires, Editorial Brujas.

Caso, Antonio, 1943, “Filósofos y moralistas franceses” $\bigotimes$, en Obras Completas, México, UNAM, 1973.

Clarín, 2012, "El psicoanálisis pierde terreno ante las ofertas 'alternativas", Buenos Aires, disponible en http://www.clarin. $\mathrm{com} /$ tendencias/psicoanalisis-pierde-terreno-ofertas-alternativas_0_750524997.htm (consultado el 15/I/2013).

Barrio Maestre, José María, 2012, Antropología del hecho religioso, Madrid, Ediciones Rialp.

Dagfal, Alejandro, S/F, "Psicología y Psicoanálisis. Entrevista a Alejandro Dagfal. Una vuelta sobre sus orígenes en París y Buenos Aires", entrevista realizada por Mónica Prandi, disponible en http://www.letraurbana.com/articulo/438 (consultado el 22/I/2013).

Deleuze, Gilles, 1983, Nietzsche et la philosophie, París, PUF.

Foucault, Michel, 2001, "Nietzsche, la généalogie, l'histoire. Hommage à Jean Hyppolite", en Dits et Ecrits II (1954-1988), Paris, Gallimard.

, 1984, "Foucault", in Huisman (D.), éd., Dictionnaire des philosophes, t. I, Paris, P.U.F., pp. 942-944.

, 1986, Historia de la sexualidad. Voluntad de saber, t.I, México, FCE.

, 1993, “QQué es la Ilustración?”, $\Delta \alpha u \mu v \eta$, Revista de Filosofía, núm. 7, pp. 5-18.

, 1996, Dits et écrits II. 1945-1969, Paris, Gallimard.

,2009, Las palabras y las cosas. Una arqueología de las ciencias humanas, México, FCE. 
Frey, Herbert, 2011, "Nihilismo y arte de la vida. Entre Montaigne y Nietzsche”, Estudios 99, vol. X, México, Instituto de Investigaciones Sociales, UNAM, invierno, pp. 33-52.

Friedrich, Nietzsche, 1996, Sobre verdad y mentira en sentido extramoral, Luis M. Valdés y Teresa Orduña (trads.), Madrid, Tecnós.

Girard, René y Gianni Vattimo, 2011, ¿Verdad o fe débil? Diálogo sobre cristianismo y relativismo, Barcelona, Paidós.

Hurtado, Guillermo, 2012, “Samuel Ramos, filósofo”, Cuadernos americanos, vol. I, México, pp. 59-69.

Le Rider, Jacques, 1999, Nietzsche en France, de la fin du XIX siècle au temps présent, Paris, PUF.

Morey, Miguel, 1990, “Introducción: la cuestión del método", en Michel Foucault, Tecnologías, del yo y otros textos afines, Barcelona, Paidós/I.C.E-U.A.B.

León, Olivé, 2007, Multiculturalismo y Pluralismo, México, Paidós/ UNAM.

Paz, Octavio, 1991, sin título, entrevista realizada por Margarita Vidal para la serie "Palabra Mayor", disponible en: http:// www.youtube.com/watch?v=Yp_xBW_TPxQ (consultado el 7/I/2013).

, 1992, El laberinto de la soledad, México, FCE.

Ramos, Samuel, 1990, "Hipótesis”, en Obras completas, vol. I, México, UNAM.

, 1990, "Perfil del hombre y la cultura en México", en Obras completas, vol. I, México, UNAM.

, 1990, Historia de la fllosofía en México, en Obras completas, vol. II, México, UNAM.

, 1962, Hacia un nuevo humanismo, México, FCE. 
,2001, Perfil del hombre y la cultura en México, Madrid, Espasa-Calpe.

Roudinesco, Elisabeth, s/f, ¿Está superado el psicoanálisis?, disponible en http://www.elortiba.org/histopsi.html (consultado el 15/I/2013).

Scott, John, 2005, Desigualdad de oportunidades y politicas públicas en México: el fracaso del proyecto redistributivo, núm. 330, México, CIDE.

Uranga, Emilio, 1952, Ontología del mexicano, México, EspasaCalpe.

Vargas Lozano, Gabriel, 2005, Esbozo histórico de la filosofía en México (siglo XX) y otros ensayos, México, Ideas mexicanas.

, 2007, "El debate filosófico de los sesenta y su significación actual", en Jorge Martínez Conteras y Aura Ponce de León, El saber filosófico. Ciencia y sociedad, vol. II, México, Siglo XXI/ AFM.

Villegas, Abelardo, 1993, El pensamiento mexicano del siglo XX, México, FCE.

Zea, Leopoldo, 2001, "Prologo", en Leopoldo Zea, Conciencia y posibilidad del mexicano/El occidente y la conciencia de Méxicol Dos ensayos sobre México y lo Mexicano, México, Porrúa.

Recibido: 17 de enero de 2014 Aceptado: 24 de noviembre de 2014 